\title{
Google Drive como entorno virtual de investigación científica para el desarrollo de la escritura académica
}

\section{Google Drive as a Virtual Scientific Research Environment for the Development of Academic Writing}

\author{
Juan Martín Ceballos Almeraya \\ Universidad Privada del Estado de México \\ Instituto Americano Cultural S. C. \\ almeraya_6@hotmail.com
}

8

ResUmen

La presente experiencia de innovación tiene el objetivo de exponer el proceso metodológico que se aplicó en el desarrollo del aprendizaje autodirigido de estudiantes de pedagogía, constituido por la aplicación de estrategias investigativas, entre éstas se encuentra un entorno virtual de investigación científica (Google Drive) que permitió no sólo la búsqueda, el manejo y el análisis de información, sino también la generación de contenido por los propios discentes a través de un análisis documental y el parafraseo de la información, como base para desarrollar una escritura académica. Para medir el impacto de la estrategia, se diseñó y se aplicó una rúbrica analítica socioformativa. Los resultados arrojados fueron positivos para su aplicación en los cuatrimestres siguientes.

Palabras clave: Aprendizaje autorregulado, entorno virtual de investigación científica, estrategias investigativas.

\section{ABSTRACT}

This innovative experience aims to share the methodology that was used in the development of Self-guided learning in students of Pedagogy. The methodology comprises the application of research strategies, including a virtual scientific research environment (Google Drive). Google Drive not only permitted one to search for, work with, and analyze information, but also allowed but also allow teachers to create content by way of document analysis and paraphrasing information, as a basis for developing academic writing. To measure the effectiveness of this strategy, an analytic socio-educational rubric was designed and applied. The results obtained favored the application of this strategy during the next academic quarters.

Keywords: Self-regulated learning, virtual scientific research environment, research strategies.

Fecha de recepción: 10/03/2019

Fecha de aceptación: 07/06/2019

14 - Google Drive como entorno virtual de investigación cientifica para el desarrollo de la escritura académica Juan Martín Ceballos Almeraya. Didac 75 (2020): 14-21 


\section{Introducción}

El desarrollo de la sociedad del conocimiento ha generado que la educación gire en torno a la producción de saberes como un valor económico, lo cual hace que la escritura científica adquiera un valor mayor que el dinero. Una prueba de ello es la creación de artículos científicos para la transacción económica en el mundo de la ciencia (Gordillo Alfonso 54). Sin embargo, al tomar en cuenta que su objetivo -el de la escritura científica- es la búsqueda, el manejo, la creación y la difusión del conocimiento como tal (Tobón et al. 20), resulta posible considerar que la formación de todo discente universitario debe integrar a la investigación como estrategia didáctica en la generación de sus conocimientos y no en la repetición de contenidos.

El trabajo que se ha realizado en dos universidades distintas de Ecatepec, Estado de México (Instituto Americano Cultural S. C. y Universidad Privada del Estado de México [upem]), ha permitido identificar algunos vacíos académicos que fracturan la formación integral, tales como: la búsqueda y el análisis de información más allá de la otorgada por los docentes, la redacción de un trabajo académico, el uso de un formato de citación -como el estilo APA- y la generación de conocimientos científicos, interpretados como contenidos del curso.

Ante esta situación, se decidió desarrollar un proyecto institucional a partir de la siguiente pregunta: ¿qué metodología podría utilizarse para que los estudiantes de pedagogía construyan sus contenidos bajo la perspectiva de aprender a aprender? Para ello se desarrolló una investigación de tipo documental de acuerdo con el estudio de categorías. El resultado fue el diseño de una metodología para aplicar la investigación como estrategia didáctica para el desarrollo de aprendizaje autorregulado.

Sin embargo, antes de su aplicación como tal, tuvo que buscarse un espacio virtual de aprendizaje colaborativo que permitiera la retroalimentación del docente, como parte de una evaluación formativa (Pasek de Pinto y Mejía 178-180), y la generación de contenido desde la misma colaboración investi- gativa. Se eligió Google Drive como entorno virtual de investigación (EVI) (Mena y Lizenberg 3).

El objetivo de dicho proyecto institucional no está enfocado sólo en su fundamentación y en la presentación de resultados de impacto del espacio virtual, sino que, de igual forma, pretende generar una reflexión del quehacer docente para establecer un vínculo entre la construcción de un conocimiento y la necesidad de difundir uno nuevo, sea escrito u oral.

\section{Desarrollo}

El proyecto institucional nació para establecer una metodología que permitiera el desarrollo de aprendizaje autorregulado a través de la aplicación de estrategias investigativas en la construcción de contenido. Así, se inició con una investigación cualitativa documental y, posteriormente, exploratoria (Reyes López et al. 43), para esclarecer algunas categorías de investigación que sirvieran de fundamento teórico.

En un segundo momento, se diseñó una rúbrica analítica socioformativa que midiera el impacto de las estrategias investigativas, específicamente el uso de Google Drive como entorno virtual de investigación científica, durante el desarrollo del manejo de información para la construcción de conocimientos, tanto de manera oral como escrita. Para comprender la experiencia de innovación, el proyecto se dividió en tres momentos: 1) la exposición de categorías que sustentaron la investigación, 2) la etapa exploratoria que inició con el diseño y la aplicación de la rúbrica de evaluación y 3) el análisis de resultados, con el fin de lograr una reflexión que generara recomendaciones específicas en el aula.

\section{Categorías de análisis}

\section{a) Aprendizaje autorregulado}

Se entiende por autorregulación del aprendizaje al proceso que facilita el desarrollo de la competencia de aprender a aprender; que permite la autodirección como capacidad de transformar o construir aptitudes mentales en un contexto académico; donde se puede identificar una necesidad o au- 
sencia de saberes para dar paso al surgimiento de investigaciones que, desde las teorías cognitivas, pueden considerarse como aprendizaje activo y procesamiento de la información. Asimismo, en esta autorregulación, el alumno se encuentra en el centro de la educación como sujeto que no sólo aprende, sino que posee la capacidad de activar su consciencia para crear estrategias de resolución.

En otras palabras, este tipo de aprendizaje coloca al estudiante en el centro de su aprendizaje, a partir de la elección de sus metas, la generación de una planificación, el propio monitoreo, su regulación y control de las actividades a realizar; acciones que a su vez permiten el desarrollo de su motivación y, con ello, conductas de aprendizaje personales y de contexto (Baez y Alonso 293).

Para aprender, los estudiantes hacen uso de una gama de estrategias para regular ciertos aspectos cognitivos, motivacionales y conductuales, así como algunas características ambientales (Yot Domínguez y Marcelo 292). Por lo tanto, si el aprendizaje es un proceso que permite el desarrollo de habilidades, aprendizaje autorregulado o autorregulación así como aprendizaje autónomo, debe llevar al desarrollo habilidades cognitivas donde éstas se conciben como estrategias compuestas de operaciones cognitivas sobre los procesos involucrados en la resolución de una tarea, pudiendo organizarse en una secuencia de operaciones interdependientes bajo la persecución de objetivos cognitivos como la comprensión y la resolución de problemas, y potencialmente pueden ser conscientes y controlables (Arancibia et al. 125-126).

\section{b) Entorno virtual de investigación científica (EvI)}

Un entorno virtual de investigación científica (EVI) incluye inicialmente infraestructura computacional y de comunicación. Sin embargo, son los recursos en red los que ayudan -con el concurso de la computación distribuida- a que los científicos dispongan de una gran cantidad de datos para su trabajo a través de repositorios como Redalyc o Google Académico, y que lo hagan merced al desarrollo de herramientas en línea de manejo de contenidos y de un middleware en un marco coherente para todas las disciplinas y los tipos de investigación, con estándares de comunicación y de circulación de datos, utilizando entornos comunes en su aspecto y opciones de proceso (Mena y Lizenberg 3).

Es importante considerar que, en los proyectos de investigación, los discentes deben contar con estrategias que les permitan tener una evolución en su trabajo y, de esta manera, una mejor visión del mismo, sobre todo dentro de la comunicación, área que ha favorecido el intercambio de conocimientos por la red. Una de estas estrategias es Google Drive como EVI, por ser una aplicación tipo Word, pero en línea, que permite el trabajo colaborativo en el desarrollo de proyectos educativos, tanto de forma sincrónica como asincrónica. Ya sea que el trabajo se realice desde un dispositivo móvil o una computadora fija o portátil, Google Drive amplía la posibilidad editar documentos las veces necesarias hasta finalizarlo según los lineamientos requeridos (Ubilla Rosales et al. 334-335).

c) Google Drive como entorno virtual de investigación científica

Google Drive es una aplicación que forma parte de la plataforma gratuita Google Apps; está disponible en la red, localizada en la nube y tiene un espacio de almacenamiento variable desde $5 \mathrm{~GB}$, con la posibilidad de aumentarlo a 15 GB si se dispone de una cuenta de Gmail, e incluso se puede obtener más espacio si se abona una cantidad mensual. Por estas razones, Google Drive se tomó como propuesta para el desarrollo de los proyectos de investigación, pues no sólo se considera como estrategia, sino también como medio para la divulgación de conocimientos entre distintos miembros del equipo, del grupo y del docente encargado, propiciando la participación grupal y colaborativa. Si se tiene en cuenta la importancia que posee el trabajo en equipo dentro de los proyectos de investigación, Google Drive permite la interacción constante entre discentes y entre docentes y estudiantes.

Google Drive favorece el trabajo colaborativo que puede realizarse en los ambientes virtuales, ya que posibilita que toda persona, aunque no coincida

16 - Google Drive como entorno virtual de investigación cientifica para el desarrollo de la escritura académica Juan Martín Ceballos Almeraya. Didac 75 (2020): 14-21 
en tiempo y espacio, converja para un fin común, que en este caso es la construcción continua del objeto de estudio de la investigación, por medio de un soporte tecnológico, tanto en espacios sincrónicos como asincrónicos, donde éste último es el más importante, dado que a veces resulta complejo organizar chats grupales por las múltiples obligaciones de los estudiantes y trabajadores.

Desde el punto de vista colaborativo, el manejo de Google Drive promueve el logro de objetivos cualitativamente ricos en contenido, pues reúne propuestas y soluciones de varias personas del grupo. Asimismo, esta estrategia permite que: 1) se valore el conocimiento de los demás miembros; 2) que se incentive el desarrollo del pensamiento crítico y la apertura mental; 3) que se conozcan diferentes temas y se adquiera nueva información; y 4) que se fortalezca el sentimiento de solidaridad y respeto mutuo, basado en los resultados del trabajo en grupo.

Por supuesto, durante este proceso es de suma importancia el acompañamiento del docente para verificar las entradas y aportaciones que hace cada uno de los integrantes del equipo en pro de su proyecto, las cuales tendrán repercusiones en su calificación parcial, como lo estipula la institución (Mora Vicarioli y Hooper Simpson 4-6).

\section{d) Metodología didáctica}

Los grupos en los que se aplicó la estrategia fueron dos, correspondientes a las siguientes universidades:
Instituto Americano Cultural S. C. y UPEM, ambas ubicadas en Ecatepec, Estado de México (ver Tabla 1).

La metodología utilizada para el desarrollo del aprendizaje autorregulado se basó en cuatro etapas: 1) el desarrollo de la UVE socioformativa, que consiste en organizar información a través de este gráfico, la cual permite identificar necesidades de conocimientos o la resolución de un problema (en este caso educativo); 2) la planeación de objetivos, metas, categorías de análisis, así como el encuadre de la investigación desde una postura colaborativa, responsable y metacognitiva (Bermeo et al. 109; González Peña 135) para la organización de ideas previo a la investigación; 3) el uso de Google Drive para el desarrollo de la investigación con base en la UVE; primero como análisis documental (Hernández Ayala y Tobón Tobón 401; Ortega Carbajal et al. 143) y, posteriormente, para el parafraseo de los conceptos que componen el contenido del tema a investigar; y 4) la retroalimentación a los estudiantes durante el proceso de las tres etapas anteriores, ya sea de forma presencial (sincrónica) o virtual (asincrónica). Cabe destacar que esta última etapa se realiza de manera transversal (ver tabla 2).

El proceso fue de la siguiente manera:

I. Formación de grupos de entre dos y cuatro personas; repartición de temas; realización y explicación de la UVE socioformativa.

Tabla 1. Grupos Participantes

\begin{tabular}{c|c|c}
\hline Curso i9/I & Instituto Americano Cultural S. C. & UPEM \\
\hline Cuatrimestre & $7 . \mathbf{0}$ & $4 . \mathbf{0}$ \\
Total de estudiantes & 16 & 19 \\
Asignatura & Taller de Psicopedagogía II & Medios y Recursos Educativos \\
\hline
\end{tabular}

Tabla 2. Metodología didáctica

\begin{tabular}{l|c|c}
\hline Uve socioformativa & Análisis documental en Google Drive & Redacción en Google Drive \\
\hline \multicolumn{3}{c}{ Realimentación y explicación de los avances de investigación } \\
\hline
\end{tabular}




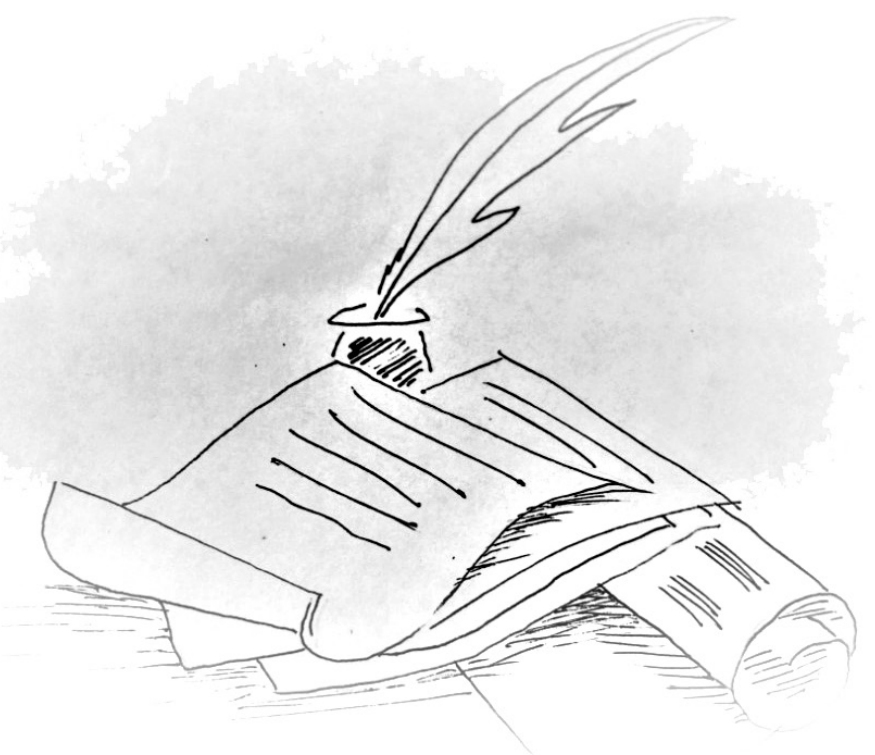

2. Creación de documento en Google Drive para iniciar el análisis documental. Los pasos en esta fase fueron los siguientes:

a) Búsqueda de información. Al identificar la información de cada categoría, se copió y pegó en el apartado correspondiente, de modo que se debía obtener, al final, por lo menos cinco conceptos de autores distintos. Todo esto se transcribió entre comillas, se consignó el número de página, se escribió la cita y las referencias en orden alfabético de acuerdo con el estilo APA.

b) La búsqueda de información en Google Académico y Redalyc a partir de la guía del docente. En esta etapa se expusieron los avances de la investigación, pues la parte oral manifestó el desarrollo de habilidades cognitivas, como la creatividad y la metacognición (Arancibia et al. 132-141).

3. Construcción conceptual del contenido, donde se retomaron los términos que conformaron las distintas categorías, se copiaron y pegaron consecutivamente con citas, se identificaron las ideas clave, se eliminaron las palabras sobrantes $y$, entonces, se unieron las ideas centrales a partir de lo ya construido mentalmente por el discente durante el análisis documental. De modo que para construir la categoría (concepto, proceso, características, elementos) se resguardaron las ideas centrales de los autores y las citas correspondientes. Asimismo, se ordenaron las referencias de consulta bajo los lineamientos del estilo APA. Cabe destacar que en esta parte del proceso fue indispensable el parafraseo. Después volvieron a exponerse los avances de la investigación en el aula, como parte de la metodología de clase bajo la realimentación del docente.

\section{Etapa exploratoria}

a) Diseño de rúbrica analítica

La concepción de la metodología aquí explicada conllevó el diseño de una rúbrica analítica socioformativa, con el propósito de integrar la vivencia del trabajo colaborativo y ético, al desarrollo de la metacognición, a la autonomía y a la resolución de problemas reales (Hernández Mosqueda et al. 360); muy acorde con el trabajo en Google Drive. Así, se organizó en 11 ítems, enmarcados en cinco dimensiones (búsqueda, organización, análisis de información, generación de conocimientos y uso de citación estilo APA), y en cuatro niveles de dominio: receptivo, resolutivo, autónomo y estratégico.

18 - Google Drive como entorno virtual de investigación cientifica para el desarrollo de la escritura académica Juan Martín Ceballos Almeraya. Didac 75 (2020): 14-21 


\section{Análisis de datos}

Para esta fase exploratoria, se obtuvieron los resultados de mayor frecuencia, representados en porcentajes para su mejor comprensión (ver Tablas 3 y 4).

Los resultados generales arrojan que la mayoría de los estudiantes se encuentran en los dominios de autonomía y de estrategia, lo cual significa que son capaces de buscar, analizar e interpretar información desde el punto de vista colaborativo. Asimismo, indagan la forma de resolver conflictos durante el proceso de investigación para alcanzar sus metas expositivas y de escritura, sobre todo, en el planear, examinar, tomar decisiones y resolverlas. Esto les permitió identificar aciertos, errores, limitaciones, así como apreciar sus logros como equipo (Roa Ordóńez 209-210).

Es importante señalar que aun cuando los discentes reflejan su ubicación dentro de los dos valores más altos, en el Instituto Americano Cultural S. C. se trabajó directamente en laboratorio de cómputo, mientras que en la UPEM no: cada estudiante lo hacía desde su casa, trabajo o celular; incluso las realimentaciones se llevaban a cabo de manera sincrónica dentro del documento de Google Drive o en el aula con la computadora portátil del docente.

Tabla 3. Frecuencia Instituto Americano Cultural S. C.

\begin{tabular}{|c|c|c|c|c|}
\hline İTEM & VALOR O & VALOR I & VALOR 2 & VALOR 3 \\
\hline 1. Organización de tabla de contenido & & & $65 \%$ & $35 \%$ \\
\hline 2. Organización de información & & & $65 \%$ & $35 \%$ \\
\hline 3. Búsqueda de información & & & $65 \%$ & $35 \%$ \\
\hline 4. Análisis de información & & & $65 \%$ & $35 \%$ \\
\hline 5. Síntesis de información (parafraseo) & & & $65 \%$ & $35 \%$ \\
\hline 6. Construcción de conocimientos & & & $65 \%$ & $35 \%$ \\
\hline 7. Planificación de investigación & & & $65 \%$ & $35 \%$ \\
\hline 8. Toma de decisiones & & & $65 \%$ & $35 \%$ \\
\hline 9. Citación con estilo APA & & & $65 \%$ & $35 \%$ \\
\hline 10. Integración de conocimientos & & & $65 \%$ & $35 \%$ \\
\hline 11. Autoaprendizaje & & & $65 \%$ & $35 \%$ \\
\hline
\end{tabular}

Tabla 4. Frecuencia UPEM

\begin{tabular}{|c|c|c|c|c|}
\hline ÍTEM & VALOR O & VALOR I & VALOR 2 & VALOR 3 \\
\hline 1. Organización de tabla de contenido & & $30 \%$ & $60 \%$ & $10 \%$ \\
\hline 2. Organización de información & & & $60 \%$ & $40 \%$ \\
\hline 3. Búsqueda de información & & & $70 \%$ & $30 \%$ \\
\hline 4. Análisis de información & & $15 \%$ & $75 \%$ & $15 \%$ \\
\hline 5. Síntesis de información (parafraseo) & & $20 \%$ & $60 \%$ & $20 \%$ \\
\hline 6. Construcción de conocimientos & & $5 \%$ & $40 \%$ & $55 \%$ \\
\hline 7. Planificación de investigación & & $5 \%$ & $55 \%$ & $40 \%$ \\
\hline 8. Toma de decisiones & & $15 \%$ & $35 \%$ & $50 \%$ \\
\hline 9. Citación con estilo APA & & $15 \%$ & $55 \%$ & $30 \%$ \\
\hline 10. Integración de conocimientos & & $15 \%$ & $75 \%$ & $10 \%$ \\
\hline 11. Autoaprendizaje & & $10 \%$ & $60 \%$ & $30 \%$ \\
\hline
\end{tabular}


Los resultados obtenidos van dirigidos hacia la construcción y reconstrucción de la realidad mediante la generación de hipótesis personales que se confrontan con otros actores del mismo nivel cognitivo. Así, se conjugan las experiencias previas con motivaciones y actitudes propias, enmarcándose en una relación con los textos, con un nuevo vocabulario, una nueva gramática y serie de estructuras gráficas (Ballesteros 444), las cuales dan pie a la interpretación de este proceso de comunicación, ya sea escrito u oral. Se fortaleció la responsabilidad y el compromiso para lograr una mejor comprensión del proceso de la escritura desde la revisión y crítica de los textos, no únicamente de forma individual, sino de igual manera entre pares (Márquez Guzmán y Gómez Zermeño 1-2). Esto conlleva a que los no especialistas científicos, en este caso estudiantes de licenciatura, tomen en cuenta que la escritura es mediadora para comprender los términos técnicos desconocidos para acercarse al mundo de la ciencia.

\section{Conclusión}

Establecer un vínculo entre el aprendizaje autorregulado y algunas estrategias de investigación -principalmente en Google Drive, como en este caso- aproxima al estudiante a la búsqueda de soluciones ante un problema, ya sea cognitivo, práctico o empírico. Esto, a su vez, permite el desarrollo de habilidades y actitudes para la lectura, la escritura, así como para el pensamiento crítico, el análisis, la síntesis, el trabajo autónomo y colaborativo (Rivadeneira Rodríguez y Silva Bustillos 6-7).

Durante este proceso, se salvaguardan dos cuestiones esenciales:

1) El soporte al que se acude para la búsqueda de información -que en este caso se refiere a usuarios digitales completamente- y la tipología de herramientas tecnológicas que se emplean para la búsqueda (Casanovas 85 ).

2) El papel de la escritura como una forma de estructurar el pensamiento, el cual se transforma para manifestar, representar y transmitir contenidos, como efecto final en el proceso de aprendizaje. Esto trae consigo la producción, la recolección y organización del conocimiento, donde se desarrollan al unísono lo científico y la escritura (Giraldo 71).

Por lo tanto, la escritura académica no puede ser más que el resultado de una praxis continua (Bassi Follari 142) dentro del proceso de formación de todo estudiante a nivel superior, donde interactúan integralmente la conceptualización y representación de la tarea, los saberes previos del estudiante investigador, las exigencias del texto y todos los demás textos precedentes a la investigación como tal (Rodríguez Hernández y Leal Vera 233).

REFERENCIAS

Arancibia, Violeta, et al. Manual de psicología educativa. Chile: Ediciones Universidad Católica de Chile, 2009. Impreso.

Baez Estradas, Mario, y Jesús Alonso Tapia. "Training Strategies for Self-Regulating Motivation and Volition: Effect on Learning." Anales de Psicología 33, 2 (2017): 292-300. Web. 17 Septiembre 2018. Recuperado de: Redalyc.org

Ballesteros Pérez, Dalia Vanessa. "El lenguaje escrito como canal de comunicación y desarrollo humano." Razón y Palabras 20, 93 (2016): 442-455. Web. 03 Marzo 2019. Recuperado de: Redalyc.org

Bassi Follari, Javier Ernesto. "La escritura académica: 14 recomendaciones prácticas." Athenea Digital. Revista de Pensamiento e Investigación Social 17, 2 (2017): 95-147. Web. 03 Marzo 2019. Recuperado de: Redalyc.org

Bermeo, Yaffar, et al. "Análisis documental de la V heurística mediante la cartografía conceptual." Ra Ximhai 12, 6 (2016): 103-121. Web. 10 Julio 2018. Recuperado de: Redalyc.org
Casanovas Catalá, Montserrat. "Las herramientas 2.0 en la escritura académica: buscadores y diccionarios." Revista Folios 43 (2016): 77-88. Web. 05 Marzo 2019. Recuperado de: Redalyc.org

Giraldo Giraldo, Camilo. "Ciencia, tecnología y escritura: el encuadre científico de la expresión escrita.” Praxis \& Saber 6, 11 (2015): 67-86. Web. 05 Marzo 2019. Recuperado de: Redalyc.org

González Peña, María de Lourdes. "Uve socioformativa: estrategia didáctica para evaluar la pertinencia de la solución a problemas de contexto." IE Revista de Investigación Educativa de la Rediech 9, 16 (2018): 133-153. Web. 18 Julio 2018. Recuperado de: Redalyc.org

Gordillo Alfonso, Adriana. "La escritura científica: una revisión temática signo y pensamiento.” Signo y Pensamiento 36, 71 (2017): 52-64. Web. 06 Marzo 2019. Recuperado de: Redalyc.org

20 - Google Drive como entorno virtual de investigación cientifica para el desarrollo de la escritura académica Juan Martín Ceballos Almeraya. Didac 75 (2020): 14-21 
Hernández Ayala, Hernán, y Sergio Tobón Tobón. "Análisis documental del proceso de inclusión en la educación." Ra Ximhai 12, 6 (2016): 399-420. Web. 15 Junio 2018. Recuperado de: Redalyc.org

Hernández Mosqueda, José Silvano, et al. "Hacia una evaluación integral del desempeño: las rúbricas socioformativas." Ra Ximhai 12, 6 (2016): 359-376. Web. 17 Junio 2018. Recuperado de: Redalyc.org

Márquez Guzmán, Saraí, y Marcela Georgina Gómez Zermeño. "Grupo virtual de escritura académica. Una e-innovación para impulsar la publicación científica.” Revista Mexicana de Investigación Educativa 23, 6 (2018): 1-28. Web. 05 Marzo 2019. Recuperado de: Redalyc.org

Mena, Marta, y Nora Lizenberg. "Desarrollo de competencias investigadoras en la sociedad red." RED. Revista de Educación a Distancia 38 (2013): 1-10. Web. 15 Junio 2018. Recuperado de: Redalyc.org

Mora Vicarioli, Francisco, y Carlene Hooper Simpson. “Trabajo colaborativo en ambientes virtuales de aprendizaje: algunas reflexiones y perspectivas estudiantiles." Revista Electrónica Educare 20, 2 (2016): 1-26. Web. 12 Oct 2018. Recuperado de: Redalyc.org

Ortega Carbajal, María Fabiola, et al. "Análisis documental de la gestión del conocimiento mediante la cartografía conceptual." Ra Ximhai 11, 4 (2015): 141-160. Web. 16 Mayo 2018. Recuperado de: Redalyc.org

Pasek de Pinto, Eva, y María Teresa Mejía. "Proceso general para la evaluación formativa del aprendizaje." Revista Iberoamericana de Evaluación Educativa 10, 1 (2017): 177-193. Web. 12 Septiembre 2018. Recuperado de: Redalyc.org
Reyes López, Octavio, et al. Metodología de investigación para cursos en línea. Enciclopedia Virtual Eumed.net. Web. 12 Junio 2017. Recuperado de: https://goo.gl/CPgGfF

Rivadeneira Rodríguez, Elmina Matilde, y Ricardo José Silva Bustillos. "Aprendizaje basado en la investigación en el trabajo autónomo y en equipo.” Negotium 13, 38 (2017): 5-16. Web. 23 Septiembre 2018. Recuperado de: Redalyc.org

Roa Ordóńez, Henry. "Estrategias creativas y metacognitivas en el aprendizaje musical." Civilizar. Ciencias Sociales y Humanas 16, 30 (2016): 207-222. Web. 20 Octubre 2018. Recuperado de: Redalyc.org

Rodríguez Hernández, Blanca Araceli, y Rogelio Alberto Leal Vera. "La escritura académica en los posgrados profesionalizantes para maestros de educación básica." CPU-e, Revista de Investigación Educativa 24 (2017): 224-239. Web. 05 Marzo 2019. Recuperado de: Redalyc.org

Tobón, Sergio, et al. "Sociedad del conocimiento: estudio documental desde una perspectiva humanista y compleja." Revista Paradigma 36, 2 (2015): 7-36. Web. 23 Mayo 2017. Recuperado de: Scielo

Ubilla Rosales, Lucía, et al. "Escritura colaborativa de textos argumentativos en inglés usando Google Drive." Estudios Pedagógicos 4, 1 (2017): 331-348. Web. 20 Nov 2018. Recuperado de: Redalyc.org

Yot Domínguez, Carmen, y Carlos Marcelo. "University Students' Self-Regulated Learning Using Digital Technologies." International Journal of Educational Technology in Higher Education 14, 1 (2017): 1-18. Web. 20 Nov 2018. Recuperado de: Redalyc.org

BiBLIOGRAFÍA RECOMENDADA

Martín Roda, Eva, y Silvana Sassano Luiz. "Posibilidades de Google Drive para la docencia a distancia y en el aula." Revista Didáctica Geográfica 16 (2015): 203-220. Web. 12 Agosto 2018. Recuperado de: Redalyc.org

Orozco Valerio, María de Jesús. "La investigación como estrategia formativa." Investigaciones Andina 18, 32 (2016): 1-7. Web. 18 Julio 2018. Recuperado de: Redalyc.org

Rodríguez, Dominga Ernestina, et al. "Elementos para escribir y publicar un artículo científico en las ciencias veterinarias." REDVET. Revista Electrónica de Veterinaria 17, 4 (2016): 1-11. Web. 17 Julio 2018. Recuperado de: Redalyc.org

Silva Marini, Janete Aparecida da, y Evely Boruchovitch. "Self-Regulated Learning in Students of Pedagogy". Pai- déia 24, 59 (2014): 323-330. Web. 21 Septiembre 2018. Recuperado de: Redalyc.org

Watanabe, Graciella, y María Regina Kawamura. "El papel de la divulgación científica realizada por científicos en la formación de profesores." Revista Electrónica Interuniversitaria de Formación del Profesorado 19, 2 (2016): 61-73. Web. 23 Julio 2017. Recuperado de: Redalyc.org

Zapata Custodio, Fanny Fabiola, y Ricardo Jiménez Hernández. "Como escribir documentos científicos. Articulo Original." Salud en Tabasco 20, 1 (2014): 21-23. Web. 24 Julio 2017. Recuperado de: Redalyc.org

\section{SemblanZa}

Doctorando en Socioformación y Sociedad del Conocimiento por la Universidad Privada del Estado de México/Instituto Americano Cultural S. C., maestro en Psicología Educativa y Licenciado en Educación. Es profesor de educación básica y en educación superior imparte materias en las áreas de Pedagogía y Psicología. Diseña estrategias didácticas para la mejora del proceso de enseñanza-aprendizaje. 